\title{
EFEITO RESIDUAL DO MÉTODO FNP SOBRE O DESEMPENHO DE RESISTÊNCIA DE FORÇA NO SUPINO HORIZONTAL
}

\author{
RESIDUAL EFFECT OF THE PNF METHOD ON THE STRENGTH \\ RESISTANCE PERFORMANCE IN BENCH PRESS
}

\section{EFECTO RESIDUAL DEL MÉTODO FNP SOBRE EL RENDIMIENTO DE FUERZA RESISTENCIA EN EL PRESS DE BANCA HORIZONTAL}

\author{
Lucas Marotta Rodrigues \\ https://orcid.org/0000-0002-7788-2422 \\ http://lattes.cnpq.br/9264097671910522 \\ Universidade Federal de Ouro Preto (Ouro Preto, MG - Brasil) \\ lucas_marotta@hotmail.com \\ Renato Melo Ferreira \\ https://orcid.org/0000-0003-0944-6730 ii \\ http://lattes.cnpq.br/4325859064183464 \\ Universidade Federal de Ouro Preto (Ouro Preto, MG - Brasil) \\ renato.mf@hotmail.com \\ Bruno Ocelli Ungheri \\ https://orcid.org/0000-0003-4827-5874 (iD \\ http://lattes.cnpq.br/0701435130704078 \\ Universidade Federal de Ouro Preto (Ouro Preto, MG - Brasil) \\ bruno.ungheri@ufop.edu.br \\ Everton Rocha Soares \\ https://orcid.org/0000-0002-7765-5828 \\ http://lattes.cnpq.br/3105616681611470 \\ Universidade Federal de Ouro Preto (Ouro Preto, MG - Brasil) \\ everton@ufop.edu.br
}

\section{Resumo}

O alongamento feito antes de exercícios resistidos pode reduzir a força do músculo alongado. Objetivou-se mensurar o efeito do método Facilitação Neuromuscular Proprioceptiva (FNP) sobre o desempenho de resistência de força no supino horizontal $(\mathrm{SH})$. Avaliou-se o número de repetições máximas (nRM) e a percepção subjetiva do

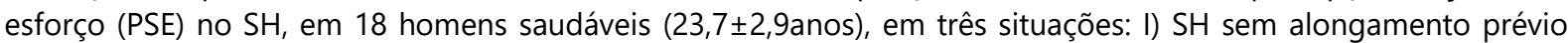
(controle); II) Alongamento FNP+SH após alongamento (FNP+SH); III) Alongamento FNP+5 minutos de intervalo $+\mathrm{SH}\left(\mathrm{FNP}+5^{\prime}+\mathrm{SH}\right)$. O valor adotado para $\mathrm{p}$ foi $<0,05$. O nRM na situação $\mathrm{FNP}+\mathrm{SH}$ foi menor do que na controle. Não houve diferenças entre o nRM da situação controle e da FNP+5'+SH, assim como entre FNP+SH e FNP+ $5^{\prime}+\mathrm{SH}$. Adicionalmente, não houve diferença na PSE nas situações avaliadas. Conclui-se que o alongamento FNP pode reduzir o desempenho de força por até cinco minutos quando aplicado imediatamente antes do exercício $\mathrm{SH}$.

Palavras-Chave: Alongamento; Resistência de Força; Supino Horizontal.

\section{Abstract}

Stretching done before resistance exercises can reduce the strength of stretched muscle. The objective was to measure the effect of the Proprioceptive Neuromuscular Facilitation (PNF) method on the strength resistance performance in bench press (BP). The number of maximum repetitions (nMR) and subjective perception of effort (SPE) in BP were evaluated in 18 healthy men (23.7 \pm 2.9 years), in three situations: I) BP without previous stretching 
(control); II) PNF+BP performed after stretching (PNF+BP); III) PNF +5 minutes of interval $+\mathrm{BP}\left(\mathrm{PNF}+5^{\prime}+\mathrm{BP}\right)$. The value adopted for $p$ was $<0.05$. The $n M R$ in $P N F+B P$ situation was lower than control. There were no differences between the $\mathrm{nMR}$ of control situation and $\mathrm{PNF}+5^{\prime}+\mathrm{BP}$, as well as between $\mathrm{PNF}+\mathrm{BP}$ and $\mathrm{PNF}+5^{\prime}+\mathrm{BP}$. Additionally, there was no difference in SPE in the situations evaluated. It is concluded that PNF stretching can reduce strength performance for up to five minutes when applied immediately before BP exercise.

Keywords: Stretching; Endurance Strength; Bench Press.

\section{Resumen}

El estiramiento realizado antes de los ejercicios de fuerza puede reducir la fuerza del músculo estirado. El objetivo fue medir el efecto del método de Facilitación Neuromuscular Propioceptiva (FNP) sobre el desempeño de fuerza resistencia en el press de banca $(\mathrm{PB})$. Se evaluó el número de repeticiones máximas (nRM) y la percepción subjetiva

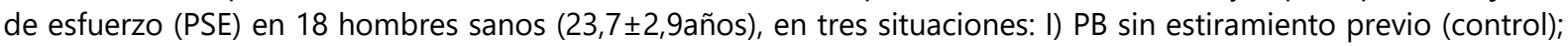
II) Estiramiento FNP+PB (FNP+PB); III) Estiramiento FNP+5 minutos + PB (FNP+5'+PB). El valor de $p$ fue $<0,05$. La $n R M$ en la situación FNP+PB fue menor que en el control. No hubo diferencias entre el nRM de la situación de control y el FNP+5'+PB, así como entre FNP+PB y FNP+5'+PB. Además, no hubo diferencia en PSE. Se concluye que el estiramiento FNP puede reducir el rendimiento de la fuerza hasta por cinco minutos cuando se aplica inmediatamente antes del PB.

Palabras clave: Estiramiento; Fuerza Resistencia; Press de Banca Horizontal.

\section{INTRODUÇÃO}

Os programas de treinamento físico comumente incluem exercícios resistidos e de flexibilidade como forma de melhorar a saúde e/ ou o condicionamento físico de seus praticantes (NÚÑEZ et al., 2018, GARBER et al., 2011, AMIRI-KHORASANI; ABU OSMAN; YUSOF, 2011). No entanto, tem sido observado que os métodos de alongamento estático passivo e Facilitação Neuromuscular Proprioceptiva (FNP), quando empregados antes dos exercícios resistidos, podem causar efeito deletério na força muscular produzida, quando comparado com o exercício resistido realizado de forma isolada (PAULO et al., 2012; DANTAS et al., 2012; OLIVEIRA; SILVA; ALONSO, 2008).

Ao considerar o alongamento estático passivo realizado previamente a exercícios resistidos, observou-se tanto redução na manifestação da força máxima dos membros inferiores (MAREK et al., 2005; PAULO et al., 2012) como dos membros superiores (PAULO et al., 2012), quanto na resistência de força dos membros inferiores (SÁ et al., 2013; DIAS et al., 2017) como dos membros superiores (DANTAS et al., 2012; PAULO et al., 2012). De forma semelhante, o método FNP quando realizado previamente a exercícios resistidos também promoveu redução da força máxima dos membros inferiores (MAREK et al., 2005) e membros superiores (GOMES et al., 2005) e na resistência de força dos membros inferiores (GOMES et al., 2005) e dos membros superiores (FRANCO et al., 2008).

Estudos foram conduzidos com a finalidade de investigar o efeito residual deletério do alongamento estático passivo sobre a produção de força muscular. Souza, Paz e Miranda e colaboradores (2013) ao realizarem estudo com 14 voluntários treinados em treinamento de 
força, verificaram o efeito de diferentes intervalos de recuperação entre o alongamento estático passivo e o desempenho de repetições máximas em exercício para membros inferiores e superiores. Foi observado que o efeito residual deletério do alongamento estático passivo na resistência de força havia sido abolido após pelo menos cinco minutos da realização do respectivo alongamento. Adicionalmente, Dias e colaboradores (2017), ao investigarem em 22 homens treinados (em treinamento de força) o efeito de 15 e 30 minutos de intervalo de recuperação, entre o alongamento estático passivo e o desempenho de repetições máximas em três séries dos exercícios cadeira extensora, cadeira flexora e adução de quadril, observaram que o efeito residual deletério do alongamento sobre a resistência de força já não ocorria após 15 minutos de recuperação.

No entanto, pouco se sabe sobre o efeito residual do método FNP na resistência de força muscular, apontando possíveis lacunas na literatura. Desta forma, o objetivo deste estudo foi mensurar o efeito do método FNP sobre o desempenho de resistência de força muscular no exercício supino horizontal (SH).

\section{MATERIAIS E MÉTODOS}

Este estudo é do tipo transversal com ensaio experimental randomizado e cruzado. Participaram do estudo 18 indivíduos do sexo masculino, com idade de 23,7 $\pm 2,9$ anos, com $69,7 \pm 8,4 \mathrm{~kg}$ de massa corporal, $172,5 \pm 6,9 \mathrm{~cm}$ de estatura e $8,1 \pm 2,4 \%$ de gordura corporal. Os critérios para inclusão na amostra foram: a) ter idade entre 18 a 30 anos; b) não ter resposta positiva em nenhuma alternativa do PAR-Q (THOMPSON et al., 2013 ); c) não ter se exercitado 24 horas antes dos testes; d) não apresentar nenhum problema osteomioarticular que possa ser agravado pelo exercício SH; e) não estar fazendo uso de nenhum suplemento ou substância que possa alterar os resultados da pesquisa, como cafeína, álcool, suplementos alimentares contendo creatina ou outra substância que exerça influência sobre a força muscular; f) e ser praticante de treinamento de força há pelo menos seis meses, com uma frequência mínima de três vezes por semana. Este estudo foi aprovado pelo comitê de ética em pesquisa da Universidade Federal de Ouro Preto sob o parecer de número 1.626.880.

As coletas deste estudo ocorreram no Laboratório de Musculação da Escola de Educação Física da Universidade Federal de Ouro Preto (EEFUFOP). A rotina estabelecida para 
coleta ocorreu em sete dias. Todos as sete visitas ocorreram de forma não consecutiva, com intervalo de 24 a 48 horas entre elas, sendo:

Visita 1 - Mensuração da estatura e massa corporal; Mensuração da composição corporal [a densidade corporal foi estimada a partir do método de três dobras cutâneas (JACKSON; POLLOCK, 1978), utilizando-se de adipômetro científico (Cescorf ${ }^{\circledR}$, Porto Alegre). O valor de densidade corporal obtido foi convertido em percentual de gordura pela equação de Siri (1961)]; Aplicação do PAR-Q (THOMPSON et al., 2013); Padronização da execução do exercício SH, quanto ao posicionamento do corpo no aparelho, das mãos na barra, da amplitude a ser realizada (completa) e do ritmo de movimento (dois segundos para a fase excêntrica e 2 segundos para a fase concêntrica (2:2) (controlado por metrônomo digital Korg ${ }^{\circledR}$ São Paulo).

Visita 2 - Realização do teste de predição de uma repetição máxima (1-RM) (BAECHLE; GROVES, 1992), para o exercício SH [realizado no banco Righetto ${ }^{\circledR}$ (modelo FS3060, Campinas), com uma barra de 1,80 metro, pesando $12 \mathrm{~kg}$ e diferentes anilhas; este material foi o mesmo utilizado nas demais visitas].

Visitas 3 e 4 - Realização do teste de 1-RM (KRAEMER; FRY, 1995) para o exercício SH (Visita 4 foi a reprodutibilidade do teste de 1-RM), não houve controle do ritmo de execução dos voluntários para os testes de 1-RM.

Visitas 5, 6 e 7 - Voluntários foram divididos de forma randomizada em três situações: A) Exercício SH sem alongamento prévio (situação controle); B) Alongamento FNP + Exercício SH imediatamente após o alongamento $(\mathrm{FNP}+\mathrm{SH}) ; \mathrm{C})$ Alongamento FNP + 5 minutos de intervalo + Exercício SH $\left(\mathrm{FNP}+5^{\prime}+\mathrm{SH}\right)$. O intervalo foi controlado com um cronometro digital (Gonew ${ }^{\circledR}$, São Paulo). O ritmo de execução do SH foi 2:2, controlado por metrônomo digital (Korg ${ }^{\circledR}$, São Paulo). A percepção subjetiva de esforço (PSE) foi coletada imediatamente após o exercício SH, por meio da escala de OMNI-RES (ROBERTSON et al., 2003).

Para maior confiabilidade dos resultados, antes do início deste estudo, foi realizado estudo piloto de duas semanas, com cinco voluntários que atendiam os critérios de inclusão. Os voluntários do estudo piloto não fizeram parte da amostra do presente estudo. Cumpre ressaltar que este procedimento, além de otimizar a aplicação dos protocolos pelos pesquisadores envolvidos, promove o alinhamento do todo o escopo de trabalho, evitandose correções de rota após o início da coleta de dados a ser mobilizada para análise. 
A operacionalização da coleta de dados nas visitas 5, 6 e 7 ocorreu da seguinte maneira:

I. Para realização do exercício SH, o voluntário deitava-se em decúbito dorsal no banco (Righetto ${ }^{\circledR}$, modelo FS3060, Campinas), com os pés apoiados no chão e joelhos em $90^{\circ}$. A execução do movimento (repetição) era iniciada com as mãos em pronação, os ombros em adução horizontal e cotovelos estendidos (a distância entre as mãos foi padronizada de acordo com a habitualidade de cada voluntário). Em seguida o voluntário realizava a fase excêntrica (abdução horizontal completa dos ombros, até que a barra tocasse no peito, na altura do processo xifoide). A execução era completa quando o voluntário retornava a barra até a posição inicial (final da fase concêntrica).

II. Para a situação controle $(A)$, inicialmente era realizado um aquecimento específico de seis a 10 repetições, com carga equivalente a 40\% de 1-RM; após três minutos de intervalo, realizava-se mais seis a 10 repetições com carga equivalente a 50\% de 1-RM; e após cinco minutos de intervalo realizava-se uma única série com o máximo de repetições possíveis no exercício $\mathrm{SH}$ com carga equivalente a 80\% de 1-RM.

III. Na situação $F N P+S H(B)$, tal como na situação $A$, os voluntários realizaram aquecimento com 40 e $50 \%$ de 1-RM; após cinco minutos de intervalo aplicou-se o método FNP, seguido imediatamente da realização do máximo de repetições possíveis no $\mathrm{SH}$, com carga equivalente a $80 \%$ de $1-\mathrm{RM}$.

IV) Para a situação FNP+5'+SH (C), tal como ocorrido nas situações $A$ e $B$, foram realizados os aquecimentos com 40 e 50\% de 1-RM; aplicou-se o método FNP, aguardou-se cinco minutos de intervalo, realizando em seguida o máximo de repetições possíveis no SH com carga equivalente a $80 \%$ de 1-RM. A realização de dois aquecimentos prévios antes dos testes ( $A, B$ e C) foi adotada a fim de reforçar com os voluntários o ritmo de execução ditado pelo metrônomo (2:2).

V) Foi solicitado a cada voluntário, imediatamente após o término da série, de cada situação, que apontasse na escala de OMNI-RES (ROBERTSON et al., 2003) o número que representasse sua PSE. Para avaliar a PSE durante uma seção de treinamento resistido, Robertson e colaboradores (2003) desenvolveram e validaram a escala de OMNI-RES, que conta com imagens ilustrativas e uma numeração crescente as quais facilitam o processo de assimilação do esforço realizado. Tal escala é utilizada em diferentes tipos de treinamento e em grupos heterogêneos, como em idosos (GEARHART et al. 2009), em pacientes com doenças 
crônicas (MORISHITA et al. 2013) e atletas (SILVA et al., 2011), o que demonstra real aplicabilidade de tal instrumento.

A figura 1 ilustra o posicionamento dos voluntários para realização do alongamento FNP. O alongamento FNP foi aplicado conforme descrito por McAtee (2002), a partir de três passos: $1^{\circ}$ passo $=0$ avaliador mobiliza os membros superiores até o limite de amplitude do voluntário; $2^{\circ}$ passo $=$ o voluntário realiza contração isométrica máxima contra as mãos do avaliador, durante oito segundos; $3^{\circ}$ passo $=0$ avaliador solicita que 0 avaliado relaxe a musculatura dos membros superiores e faz um forçamento do movimento além do limite original. Este procedimento foi repetido por 3 vezes consecutivas.

Figura 1 - Ilustração do posicionamento para realização do alongamento FNP no peitoral maior

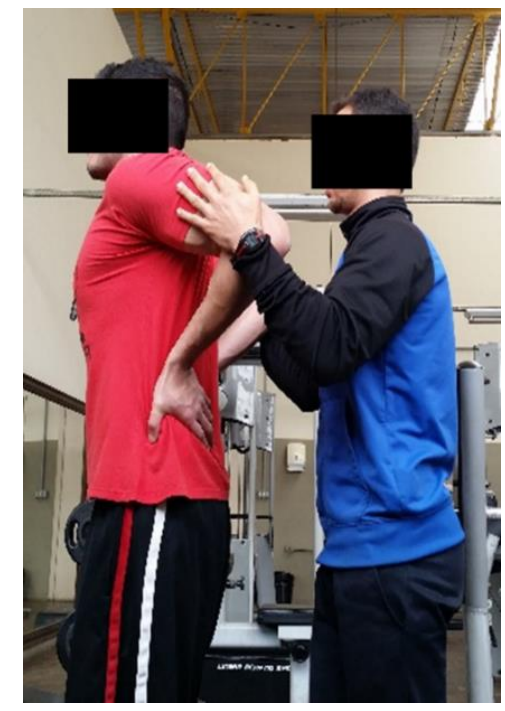

Fonte: arquivo dos autores

Os dados se apresentam em média \pm desvio padrão. Para avaliação da distribuição de normalidade dos dados foi utilizado o teste de D'agostino e Pearson. Excetuando a idade dos voluntários, foi observada distribuição de normalidade para todos os parâmetros observados ( $p>0.05$ ). Para avaliação da reprodutibilidade e correlação entre os testes de 1-RM foi utilizado o teste t pareado. Para comparação entre o número de repetições e PSE no exercício $\mathrm{SH}$, nas diferentes condições, foi utilizado o teste de ANOVA para medidas repetidas. Para avaliar a confiabilidade da estimativa do número de repetições máximas e PSE nas diferentes condições experimentais determinou-se intervalo de confiança médio de 95\% (IC 
95\%). O valor de significância foi de $p<0,05$. As análises foram realizadas pelo Software Graphpad Prism (version 5.00).

\section{RESULTADOS}

A tabela 1 apresenta os valores das cargas máximas encontradas no exercício SH no teste de predição, teste de 1-RM e a reprodutibilidade do teste de 1-RM. Não foi observada diferença significativa entre os três testes de força máxima realizados.

Tabela 1 - Valores das cargas máximas encontradas no exercício supino horizontal (SH)

\begin{tabular}{c|c|c|c}
\hline $\mathrm{n}$ & Predição 1-RM (Kg) & Teste 1-RM (Kg) & Reprodutibilidade (Kg) \\
\hline 18 & $89,0 \pm 13,0$ & $89,2 \pm 13,2$ & $89,2 \pm 12,4$ \\
\hline
\end{tabular}

Valores em média \pm desvio padrão. 1 -RM $=1$ repetição máxima. ANOVA para medidas repetidas.

Fonte: construção dos autores

A tabela 2 apresenta o número de repetições máximas executadas no exercício $\mathrm{SH}$, com $80 \%$ de 1-RM, na situação controle e naquelas onde foi empregado o método FNP $(\mathrm{FNP}+\mathrm{SH}$ e FNP +5'+SH). Pode-se observar que o número de repetições máximas foi menor na situação FNP+SH quando comparado à situação controle. No entanto, não foram observadas diferenças entre o número de repetições máximas entre a situação controle e a $\mathrm{FNP}+5^{\prime}+\mathrm{SH}$, assim como entre a situação $\mathrm{FNP}+\mathrm{SH}$ e a $\mathrm{FNP}+5^{\prime}+\mathrm{SH}$.

Tabela 2 - Número de repetições máximas (NRM) executas no exercício supino horizontal (SH)

\begin{tabular}{cccc}
\hline $\mathrm{n}=18$ & Situação Controle & $\mathrm{FNP}+\mathrm{SH}$ & $\mathrm{FNP}+5^{\prime}+\mathrm{SH}$ \\
\hline NRM & $7,6 \pm 1,3$ & $6,9 \pm 1,2^{*}$ & $7,2 \pm 1,2$ \\
IC $95 \%$ & $6,91 / 8,2$ & $6,4 / 7,5$ & $6,6 / 7,8$ \\
\hline
\end{tabular}

Valores em média \pm desvio padrão. FNP $=$ Facilitação Neuromuscular Proprioceptiva. $5^{\prime}=5$ minutos. Situação Controle $=\mathrm{SH}$ realizado sem alongamento prévio. $\mathrm{FNP}+\mathrm{SH}=\mathrm{SH}$ realizado imediatamente após aplicação do método $\mathrm{FNP}$. FNP+5 $+\mathrm{SH}=\mathrm{SH}$ realizado 5 minutos após aplicação do método FNP. IC 95\% = Intervalo de confiança médio (Inferior / Superior). $p<0,05$ em comparação com a situação controle. ANOVA para medidas repetidas seguida do pósteste de Bonferroni.

Fonte: Construção dos autores 
A tabela 3 apresenta os valores da PSE (OMNI-RES; Robertson et al., 2003) após execução de cada situação experimental no exercício $\mathrm{SH}$, observa-se que não há diferença significativa quanto à percepção subjetiva do esforço nas diferentes situações avaliadas.

Tabela 3 - Percepção subjetiva do esforço (PSE) OMNI-RES (ROBERTSON et al., 2003) no exercício supino horizontal (SH)

\begin{tabular}{cccc}
\hline $\mathrm{n}=18$ & Situação Controle & FNP + SH & FNP + 5' + SH \\
\hline PSE & $7,3 \pm 1,0$ & $7,6 \pm 1,0$ & $7,3 \pm 1,0$ \\
IC 95\% & $6,8 / 7,9$ & $7,0 / 8,1$ & $6,9 / 7,8$
\end{tabular}

Valores em média \pm desvio padrão. FNP = Facilitação Neuromuscular Proprioceptiva. $5^{\prime}=5$ minutos. Situação Controle $=\mathrm{SH}$ realizado sem alongamento prévio. $\mathrm{FNP}+\mathrm{SH}=\mathrm{SH}$ realizado imediatamente após aplicação do método $\mathrm{FNP}$. $\mathrm{FNP}+5^{\prime}+\mathrm{SH}=\mathrm{SH}$ realizado 5 minutos após aplicação do método FNP. IC 95\% = Intervalo de confiança médio (Inferior / Superior). Não houve diferença significativa $(p>0,05)$. ANOVA para medidas repetidas.

Fonte: Construção dos autores

\section{DISCUSSÃO}

No presente estudo, foi mensurado o efeito do alongamento FNP sobre o desempenho de resistência de força no exercício $\mathrm{SH}$ em indivíduos do sexo masculino fisicamente ativos. De forma geral, os dados demonstraram que, somente na situação em que o alongamento FNP foi realizado imediatamente antes do exercício $\mathrm{SH}$, observou-se uma redução do desempenho de resistência de força. No entanto, não foi verificada diferença significativa na PSE após a execução do exercício SH nas diferentes situações experimentais.

A redução na capacidade de realizar força muscular após alongamentos está relacionada a dois mecanismos principais. O primeiro mecanismo está relacionado à redução da ativação neural em resposta ao estímulo dado ao órgão tendíneo de Golgi (FOWLES; SALE; MACDOUGALL, 2000). Já o segundo mecanismo refere-se às mudanças nas propriedades mecânicas da unidade músculo-tendínea (HALAR et al., 1978). Neste caso, a tensão do tendão durante o alongamento alteraria sua viscoelasticidade, causando um comportamento de relaxamento de estresse que reduz a rigidez do tendão e afeta a força transmitida pelos músculos (TRAJANO et al., 2013). Em conjunto, esses mecanismos podem justificar o decréscimo no desempenho de resistência de força quando foi aplicado o método FNP imediatamente antes do exercício $\mathrm{SH}$. 
Adicionalmente, os resultados apresentados neste estudo corroboram aos de outros autores que identificaram redução no desempenho de força máxima (MAREK et al., 2005; GOMES et al., 2005) e de resistência de força (GOMES et al., 2005; FRANCO et al., 2008; GOMES et al., 2011) em diferentes exercícios resistidos realizados imediatamente após o alongamento FNP. Gomes e colaboradores (2011) observaram que o alongamento FNP foi capaz de reduzir o desempenho de resistência de força no exercício $\mathrm{SH}$, apenas quando este foi realizado com cargas equivalentes à 60 e 80\% de 1-RM, não sendo observado este efeito quando a carga utilizada foi de $40 \%$ de 1 -RM. Esses dados em conjunto sugerem que o efeito deletério do alongamento FNP sobre a resistência de força muscular é dependente da intensidade utilizada no exercício, ocorrendo somente em intensidades submáximas maiores do que $60 \%$ de 1-RM. Ainda, é importante considerar o trabalho realizado por Souza e colaboradores (2017) que, ao verificarem o efeito agudo do alongamento estático e o FNP no exercício SH em indivíduos treinados, perceberam que mesmo sem diferença significativa em relação ao número total de repetições e volume total, houve diminuição significativa do número de repetições entre a segunda e terceira séries, ao comparar com a primeira, nas condições apresentadas.

Souza, Paz e Miranda (2013) a fim de investigar o efeito residual do alongamento sobre a força muscular, verificaram o efeito de diferentes intervalos de recuperação entre o alongamento estático passivo e o desempenho de uma série de repetições máximas exercício voador e cadeira extensora. Foi observado que após pelo menos cinco minutos da realização do alongamento estático passivo, o efeito residual deste sobre a resistência de força muscular nos exercícios avaliados foi abolido. Apesar de o presente estudo ter utilizado o alongamento FNP, os resultados apresentam alguma semelhança quanto ao tempo de duração do efeito dos alongamentos estático passivo e FNP (até cinco minutos), sobre o desempenho de resistência de força. No entanto, é importante reforçar que em nosso estudo não foi observada diferença entre o número de repetições máximas que foram realizadas nas situações $\mathrm{FNP}+\mathrm{SH}$ e a $\mathrm{FNP}+5^{\prime}+\mathrm{SH}$. Acreditamos, que a não diferença entre essas situações (FNP+SH e a FNP+ $5^{\prime}+\mathrm{SH}$ ) possa ter ocorrido em função do tempo de intervalo adotado (cinco minutos), uma vez que Dias e colaboradores (2017) observaram que o efeito deletério do alongamento estático passivo sobre a resistência de força de exercícios de membros inferiores foi abolido após 15 minutos. Mais estudos utilizando o método FNP e diferentes intervalos de recuperação entre o alongamento e o exercício são necessários para melhor compreensão desse resultado. 
Apesar de os resultados do presente estudo apontarem diferença significativa no desempenho de resistência de força muscular comparando a situação em que se realiza o SH imediatamente após o alongamento FNP e a situação controle, essa diferença não foi observada na PSE, OMNI-RES, (ROBERTSON et al., 2003). Robertson e Noble (1997) definem a PSE como sendo a intensidade subjetiva do esforço ou fadiga que é experimentado durante a prática de atividades aeróbicas ou de força, apresentando intima relação com a medida de esforço físico. Assim é possível que os valores apontados pelos voluntários recebam alguma influência do nível de experiência que estes têm com esse tipo de instrumento, uma vez que Borg (2000) afirma existir uma parcela de indivíduos que podem apresentar dificuldades em compreender as instruções que lhes são apresentadas antes do teste, como uma dificuldade em relação as habilidades verbais e matemáticas.

Os resultados do presente estudo podem auxiliar no entendimento da influência do alongamento FNP sobre o desempenho de resistência de força muscular, bem como a duração desse efeito deletério sobre a resistência de força muscular. Entretanto, ao pensar em uma implicação prática do estudo, deve-se sempre considerar o perfil do praticante de exercícios resistidos, pensando nos seus objetivos, necessidades e limitações frente a sua periodização de treinamento. De acordo com a pesquisa em tela, o alongamento FNP teve influência deletéria sobre a resistência de força apenas quando realizado imediatamente antes do exercício, sendo significativa a exclusão de sua prática para um atleta de levantamento de peso, por exemplo; diferentemente de um indivíduo que faz seu treinamento voltado para qualidade de vida e melhora das capacidades físicas, entre elas a flexibilidade recomendando-se, portanto, a prática de exercícios de alongamento.

Algumas limitações ao estudo, são: 1- Fase de familiarização com a escala OMNIRES (ROBERTSON et al., 2003): Acreditamos que com a mudança na metodologia aplicada, aumentando-se a fase de adaptação, seria possível minimizar uma possível dificuldade de interpretar a escala; 2- Intervalo de recuperação entre o FNP e o exercício resistido - É possível que a adoção de diferentes intervalos de recuperação, sejam eles maiores e menores a cinco minutos, permitam compreender melhor sobre a duração do efeito deletério do FNP sobre a resistência de força; 3 - Por fim, entende-se a necessidade de ampliar esse estudo para populações diferentes (quanto ao sexo, idade, condição física etc.), utilizando-se ainda um número maior de exercícios, séries e diferentes intensidades 


\section{CONCLUSÃO}

De acordo com os resultados obtidos, pode-se concluir que o alongamento do tipo FNP pode reduzir o desempenho de força muscular quando aplicado imediatamente antes da prática do exercício SH em adultos jovens. No entanto, este tipo de alongamento parece não afetar a PSE, a partir da Escala de OMINI-RES nas diferentes situações avaliadas.

\section{REFERÊNCIAS BIBLIOGRÁFICAS}

AMIRI-KHORASANI, Mohammadtaghi; OSMAN, Noor A Abu; YUSOF, Ashril. Acute effect of static and dynamic stretching on hip dynamic range of motion during instep kicking in professional soccer players. The journal of strength and conditioning research, v. 25, n. 6 , p. 1647-1652, jun., 2011.

BAECHLE, Thomas R.; GROVES, Barney R. Weight training: steps to success. Champaign: Leisure Press, 1992.

BORG, Gunnar. Escalas de Borg para a dor e esforço percebido. São Paulo, SP: Manole, 2000.

DANTAS, Rogerio e colaboradores. Efeito do alongamento estático no desempenho da força. EFDeportes, v. 15, n. 166, mar., 2012.

DIAS, Henrique e colaboradores. Number of repetition after different rest intervals between static stretching and resistance training. Revista andaluza de medicina del deporte, v. 10, n. 3, p. 132-136, sep., 2017.

FRANCO, Bruno L. e colaboradores. Acute effects of different stretching exercises on muscular endurance. The journal of strength $\&$ conditioning research, v. 22, n. 6, p. 1832-1837, nov., 2008.

FOWLES, Jonathon R.; SALE, Digby G.; and MACDOUGALL, J. Duncan. Reduced strength after passive stretch of the human plantar flexors. Journal of applied physiology, v. 89, n. 3, p. 1179-1188, Sep. 2000.

GARBER, Carol Ewing e colaboradores. American College of Sport Medicine position stand. Quantity and quality of exercise for developing and maintaining cardiorespiratory, musculoskeletal, and neuromotor fitness in apparently healthy adults: guidance for prescribing exercise. Medicine \& science in sports \& exercise, v. 43, n. 7, p.1334-1359, jul., 2011.

GEARHART, Randall F. e colaboradores. Strength tracking using the OMNI resistance exercise scale in older men and women. The journal of strength \& conditioning research, v. 23, n. 3, p. 1011-5, may., 2009. 
GOMES, Tiago Matassoli e colaboradores. Efeito agudo dos alongamentos estático e FNP sobre o desempenho da força dinâmica máxima. Revista brasileira de fisiologia do exercício, v. 4, n. 1, p. 13-21, jan., 2005.

GOMES, Tiago Matassoli e colaboradores. Acute effects of two different stretching methods on local muscular endurance performance. The journal of strength \& conditioning research, $\mathrm{v}$. 25, n. 3, p. 745-752, mar., 2011.

HALAR, Eugen M. e colaboradores. Gastrocnemius muscle belly and tendon length in stroke patients and able-bodied persons. Archives of physical medicine and rehabilitation, v. 59, n. 10, p. 476-484, oct., 1978.

JACKSON, Andrew S.; POLLOCK, Michael L. Generalized equations for predicting body density for men. British journal of nutrition, v. 40, n. 3, p. 497-504, nov., 1978.

KRAEMER, William J.; FRY, Andrew C. Strength testing: development and evaluation of methodology. In: MAUD, Peter; FOSTER Carl (Eds.). Physiological assessment of human fitness. Champaign: Human Kinetics, 1995.

MAREK, Sarah M. e colaboradores. Acute effects of static and proprioceptive neuromuscular facilitation stretching on mucle strength and power output. Journal of athletic training, v. 40, n. 2, p. 94-103, jun., 2005.

MCATEE, Robert E. An overview of facilitated stretching. Journal of bodywork and movement therapies, v. 6, n. 1, p. 47-54, jan., 2002.

MORISHITA, Shinichiro e colaboradores. Rating of perceived exertion for quantification of the intensity of resistance exercise. International journal of physical medicine $\boldsymbol{\&}$ rehabilitation, v. 1, n. 9, p. 172, jan., 2013.

NÚÑEZ, Francisco Javier e colaboradores. The effects of unilateral and bilateral eccentric overload training on hypertrophy, muscle power and COD performance, and its determinants, in team sport players. PLos one, v. 13, n. 3, p. 1-13, mar., 2018.

OLIVEIRA, Debora Perez de; SILVA, Liana Cunha da; ALONSO, Denise de Oliveira. Efeito do alongamento prévio no desempenho em teste de força muscular máxima. Revista de atenção à saúde, v. 6, n. 17, p. 54-58, jul./ set., 2008.

PAULO, Anderson Caetano e colaboradores. Efeito agudo dos exercícios de flexibilidade no desempenho de força máxima e resistência de força de membros inferiores e superiores. Motriz, v. 18 n. 2, p. 345-355, abr./ jun., 2012.

ROBERTSON, Robert J. e colaboradores. Concurrent validation of the OMNI perceived exertion scale for resistance exercise. Medicine \& science in sports \& exercise, v. 35, n. 2, p. 333-341, feb., 2003. 
ROBERTSON, Robert J.; NOBLE, BJ. Perception of physical exertion: methods, mediators, and applications. Exercise and sport sciences reviews, v. 25, p. 407-52, jan., 1997.

SÁ, Marcos André e colaboradores. Efeito agudo do alongamento estático e facilitação neuromuscular proprioceptiva sobre o desempenho do número de repetições máximas em uma sessão de treino de força. Motricidade, v. 9, n. 4, p. 73-81, dez., 2013.

SILVA, Michael Santos e colaboradores. Análise do efeito de diferentes intensidades e intervalos de recuperação na percepção subjetiva de atletas. Motricidade, v. 7, n. 1, p. 3-12, jun., 2011.

SIRI, William E. Body composition from fluid spaces and density: analyses of methods. In: BROZEK Josef; HENSCHELA, Austin (Eds.). Techniques for measuring body composition. National academy of science: Washington, 1961.

SOUZA, Jhenyffer; PAZ, Gabriel; MIRANDA, Humberto. Influência de diferentes intervalos de recuperação entre o alongamento estático passivo e desempenho de força muscular. Revista brasileira de atividade física e saúde, v. 18, n. 1, p. 86-94, mai., 2013.

SOUZA, Daniel Vieira Braña Côrtes de e colaboradores. Efeito agudo de diferentes métodos de alongamento sobre o desempenho de força em séries sucessivas. Journal of physical education, v. 28, n. 1, e2832, oct., 2017.

THOMPSON, Paul D. e colaboradores. ACSM's new preparticipation health screening recommendations from ACSM's guidelines for exercise testing and prescription. Current sports medicine reports, v. 12, n. 4, p. 215-217, jul./ aug., 2013.

TRAJANO, Gabriel S. e colaboradores. Contribution of central vs. peripheral factors to the force loss induced by passive stretch of the human plantar flexors. Journal applied physiology, v. 115, n. 2, p. 212-218, jul., 2013.

Dados do primeiro autor:

Email: lucas_marotta@hotmail.com

Endereço: Rua Doutor Cravo, 68, Tavares, Ouro Preto, MG, CEP: 35400-000, Brasil

Recebido em: 22/04/2021

Aprovado em: 26/05/2021

\section{Como citar este artigo:}

RODRIGUES, Lucas Marotta e colaboradores. Efeito residual do método FNP sobre o desempenho de resistência de força no supino horizontal. Corpoconsciência, v. 25, n. 2, p. 110-122, mai./ ago., 2021. 